\title{
Application of optimally-shaped phononic crystals to reduce anchor losses of MEMS resonators
}

\author{
R. Ardito, M. Cremonesi, L. D’Alessandro, A. Frangi \\ Politecnico of Milano \\ Dept. of Civil and Environmental Engineering \\ P.za Leonardo da Vinci 32, 20133 Milano, Italy \\ raffaele.ardito@polimi.it, massimiliano.cremonesi@polimi.it, luca.dalessandro@polimi.it, $\underline{\text { attilio.frangi@polimi.it }}$
}

\begin{abstract}
This work is focused on the application of Phononic Crystals to reduce anchor losses of MEMS contour mode resonators. Anchor losses dominates the losses in these type of released resonators at low frequency and at low temperature. The use of phononic crystals, intended as finite-periodic distribution of holes in the anchor, is fully compatible with fabrication processes and moreover it is easy to implement. The numerical results obtained in this work show how the use of these crystals can significantly reduce the anchor losses: without the use of the crystal the $Q$ factor related to only anchor losses is 344 , with the use of the crystal it can reach up to 105900 .
\end{abstract}

Keywords-MEMS resonators, anchor losses, Phononic crystals

\section{INTRODUCTION}

In the class of MEMS transducers, AlN contour mode resonators (CMRs) are widely employed for several purposes. A key parameter for assessing the figure of merit of such resonators is the quality factor Q. At low frequency (i.e. $200 \mathrm{MHz}$ ) and up to room temperature or at low temperature in general, the Q-factor is dominated by is anchor losses [1]. A valid provision to reduce anchor losses is the application of phononic crystals (PnCs), which show complete band gaps in the transmission of elastic waves, therefore they can be considered as highly efficient acoustic reflectors, which can be used in order to confine the elastic energy. The operational features of a PnC are summarized by the central frequency (which should match the resonator's properties) and the relative size of band gaps. The bang gap width is the key parameter in order to scatter the elastic waves: the wider the band gap the highest the scattering. PnCs application is already used in literature to reduce anchor losses: in [2] unreleased resonators in $\mathrm{GaN}$ are folded by solid-air PnCs, [3] presents strips built as PnCs that are placed as the anchor itself, in [4] PnCs are used to reduce anchor losses of quartz released resonators. The common feature of the PnCs described in these works is the topology of the solid-air unit cell, carachterized by a circular hole shape: this topology (see Fig. 1a) is endowed with a full bandgap of $13 \%$ gap to mid-gap ratio (see Fig. 1b) for thickness-in-plane dimensions' ratio of 0.5 and radius-unit cell carachteristic dimensions' ratio of 0.45 ; for different ratios the bandgap can be significantly reduced or disappear. This work is focused on the analysis of anchor losses in the presence of PnCs where the hole topology (see Fig. 1c) is found by means of shape optimization process exploiting the Bidirectional Evolutionary Structural Optimization technique [5]. This leads to the widest band gap of 55\% gap mid-gap ratio (see Fig. 1d) for thickness-in-plane dimensions' ratio of 0.5 , and moreover to a stronger reduction of the anchor losses by locating the PnC around the resonator's anchor. Anchor losses have been calculated simulating the dissipation of the elastic waves scattered in the substrate through the anchor using a robust three-dimensional Finite Element Model including Absorbing Boundary regions (through the Perfectly Matched Layer technique).

\section{RESONATOR ANALYSED}

The CMR resonator analysed is in AIN, $60 \mu \mathrm{m}$ wide and $148 \mu \mathrm{m}$ long, with a thickness equal to $1.2 \mu \mathrm{m}$. The natural frequency of interest is around $260 \mathrm{MHz}$ (the mode shape is

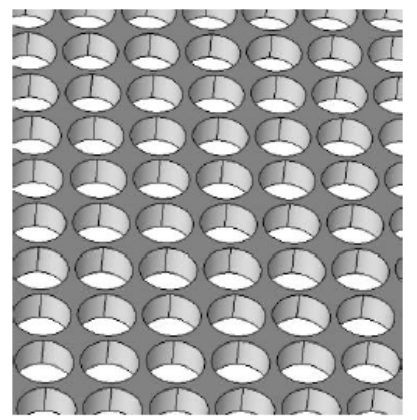

(a)

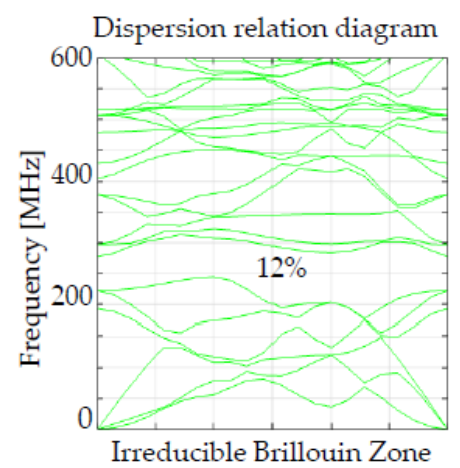

(b)

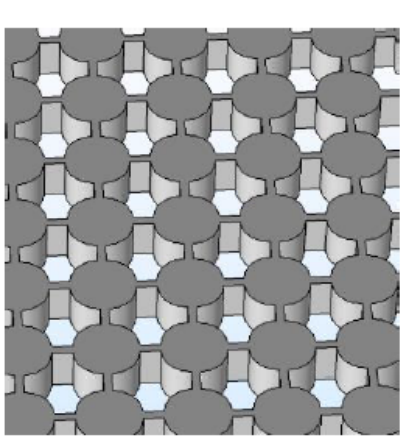

(c)

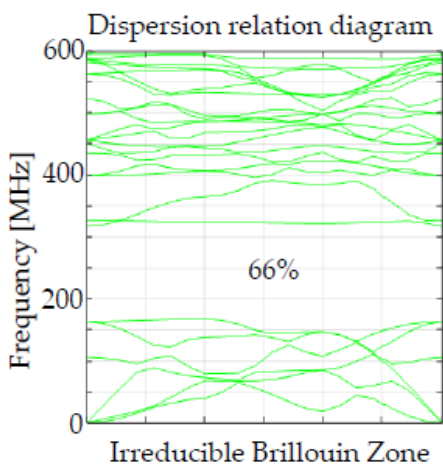

(d)

Fig. 1: Comparison between band gaps of phononic crystals with circular hole shape and optimized hole shape, the material employed is AlN. (a) and (c) represent respectively the two geometries for the infinite phononic crystal, (b) and (d) the two related dispersion relation diagrams. Details on the definition of the dispersion relation diagrams can be found in [5]. 


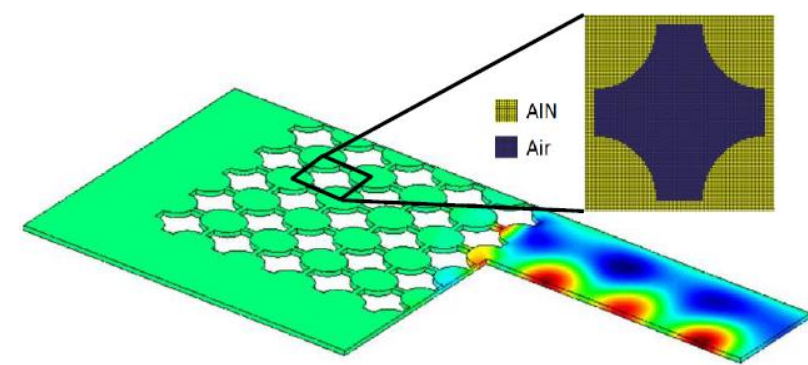

Fig. 2: Analysed CMR resonator (only $1 / 4$ of the resonator is reported): vibrational mode at $260 \mathrm{MHz}$, finite $5 \times 5 \mathrm{PnC}$ structure is placed in the anchor, the $\mathrm{PnC}$ unit cell is highlighted.

depicted in Fig. 2, where 1/4th of the CMR is drawn with the unit cell of the PnC shown in the inset). In order to match the resonant frequency of the CMR the side of the $\mathrm{PnC}$ unit cell is $14.2 \mu \mathrm{m}$ long, leading to a ratio between thickness and in-plane carachteristic dimension of the $\mathrm{PnC}$ of 0.085 . The radius of the solid circles in the optimized hole topology is about $4.7 \mu \mathrm{m}$. This optimized $\mathrm{PnC}$ is endowed with a full three-dimensional band gap for in-plane waves with a gap to mid-gap ratio of $11 \%$, centered at $260 \mathrm{MHz}$. The difference between this $11 \%$ band gap ratio and the previously calculated $66 \%$ (see Fig. 1d) is due to the different ratio between thickness and in-plane dimensions of the two PnC unit cells analysed (namely 0.085 for the first and 0.500 for the second): the smaller this ratio the narrower the bandgap. It must be noticed that for the aspect ratio of the CMR analysed here the circular hole shape with radius to in-plane carachteristic dimension ratio of 0.45 does not present any full band gap.

\section{Q-FACTOR FOR ANCHOR LOSSES USING PHONONIC CRYSTALS}

In this work several configurations of finite $\mathrm{PnC}$ are analysed numerically in terms of the resulting Q-factor for anchor losses: namely the ones depicted in Fig. 3. In Tab. 1 the Q-factor values for the configurations of Fig. 3 are reported.

\section{TABLE I. Q-ANCHOR-FACTORS FOR DIFFERENT CONFIGURATIONS}

\begin{tabular}{|l|l|l|}
\hline \multicolumn{1}{|c|}{ Configuration } & \multicolumn{1}{|c|}{ Q anchor } & Lamb mode frequency [MHz] \\
\hline (a) No PnC & 344 & 261.30 \\
\hline (b) $4 \times 1$ PnC holes & 6517 & 261.54 \\
\hline (c) $4 \times 3$ PnC holes & 13470 & 261.54 \\
\hline (d) $5 \times 3$ PnC holes & 105964 & 261.51 \\
\hline (e) $5 \times 5$ PnC holes & 105900 & 261.50 \\
\hline
\end{tabular}

The Q-factor for anchor losses only in the absence of PnC is 344 , the matching between the numerical result and the experimental testing can be found in [1]. The first comment on the results of Tab. 1 is that the use of $\mathrm{PnC}$ in the anchor does not change the resonant frequency of the CMR in all the
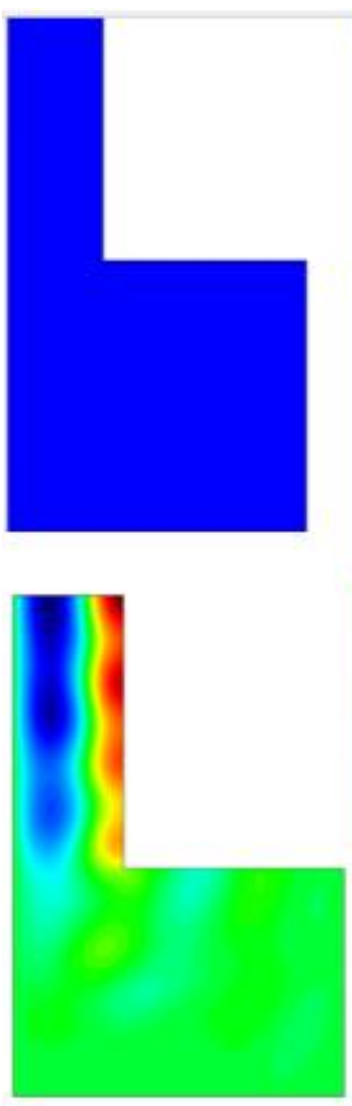

(a)
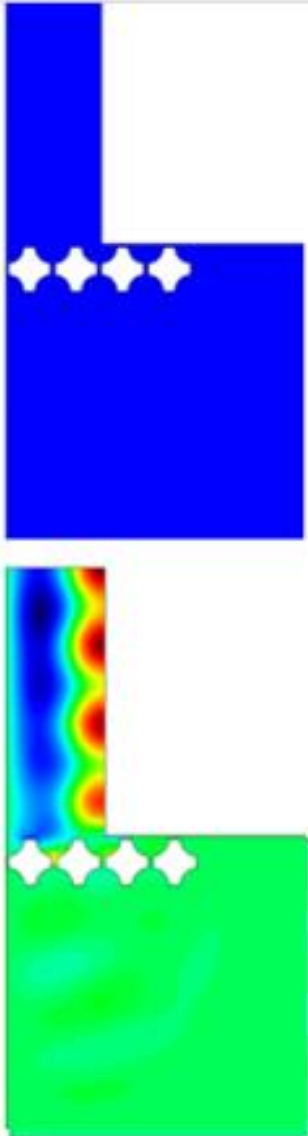

(b)
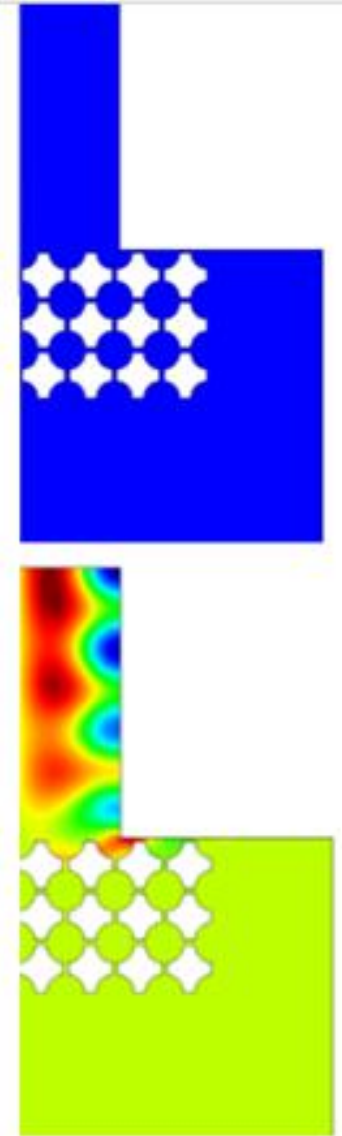

(c)
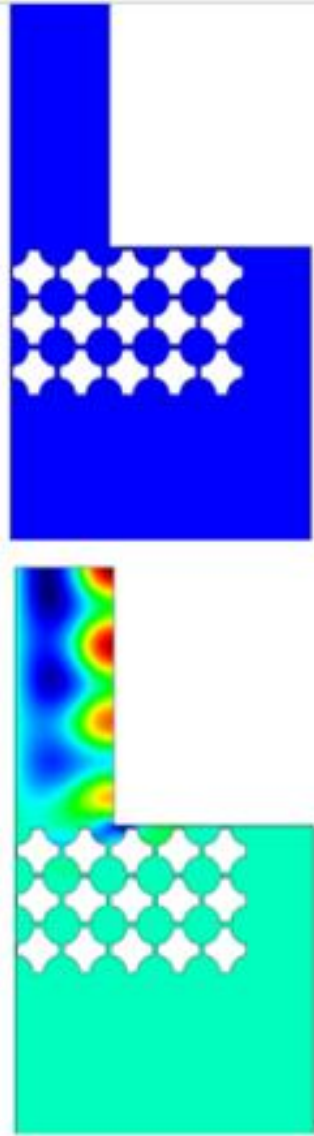

(d)
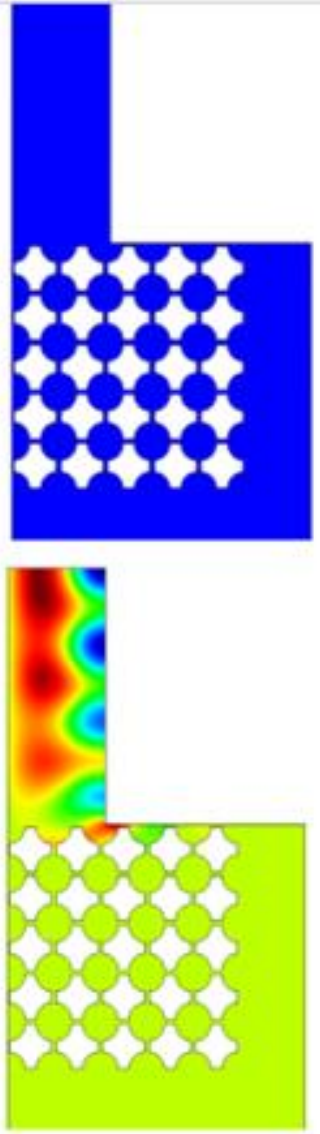

(e)

Fig. 3: Representation of a quarter of the released AIN MEMS resonator in the different configurations analysed (a)-(e). The finite phononic crystals are located in the anchor. The carachteristic mode is reported.

The quality factor for anchor losses related to the analysed mode for each of the (a)-(e) configurations are reported in Tab. 1. 
configurations considered. By inserting a single line of four PnC's unit cells the anchor losses Q-factor increases approximately by 15-20 times and it reaches the value of 6517 for the considered application. By inserting a second and a third line of four unit cells the anchor losses Q-factor reaches 13470. It must be noticed that the second main losses, namely the thermo-elastic losses, present a Q-factor around 1000020000 for the CMR analysed. In the configuration of Fig. 3d the Q-factor numerically reaches 100000 . In configuration of Fig. 3e the anchor losses Q-factor is comparable to the one of configuration (d), therefore it is possible to conclude that by inserting $5 \times 3 \mathrm{PnCs}$ finite structure the energy dissipation in the analysed CMR is no more dominated by anchor losses but by other type of losses such as thermo-elastic losses, interface losses. Further experimental investigation is required.

\section{CONCLUSIONS}

The numerical results reported in this work declare that $\mathrm{PnC}$ are good candidate for the reduction of anchor losses in released resonators. Moreover, the optimized shape presented can be easily feasible for microfabrication processes and can cancel the losses produced by the anchor if a proper configuration (e.g. the one of Fig. 3d) is adopted. In order to confirm these numerical predictions a proper experimental campaign must be conducted, nevertheless it must be noticed that the three-dimensional finite element solver used for the anchor losses calculation has been already validated in a previous work [1].

\section{REFERENCES}

[1] J. Segovia-Fernandez, M. Cremonesi, C. Casella, A. Frangi, G. Piazza, "Anchor losses in AIN contour mode resonators", Journal of microelectromechanical systems, vol. 24 no. 2, April 2015

[2] S. Wang, L.C. Popa, D. Weinstein, "GaN MEMS resonator using a folded phononic crystal structure", MIT, 2015

[3] D. Feng, D. Xu, G. Wu, B. Xiong, Y. Wang, "Phononic crystal strip based anchor for reducing anchor loss of micromechanical resonators", Journal of Applied Physics 115 024503, 2014

[4] Y.Y Chen, T.-T. Wu, S.-Y. Pao, "Anchor loss reduction of quartz resonators utilizing phononic crystals", IEEE Ultrasonic Symposium 2015

[5] L. D’Alessandro, B. Bahr, L. Daniel, D. Weinstein, R. Ardito, "BESO approach to topology optimization of GaN phononic crystals" ECCOMAS 2016, Crete Island 